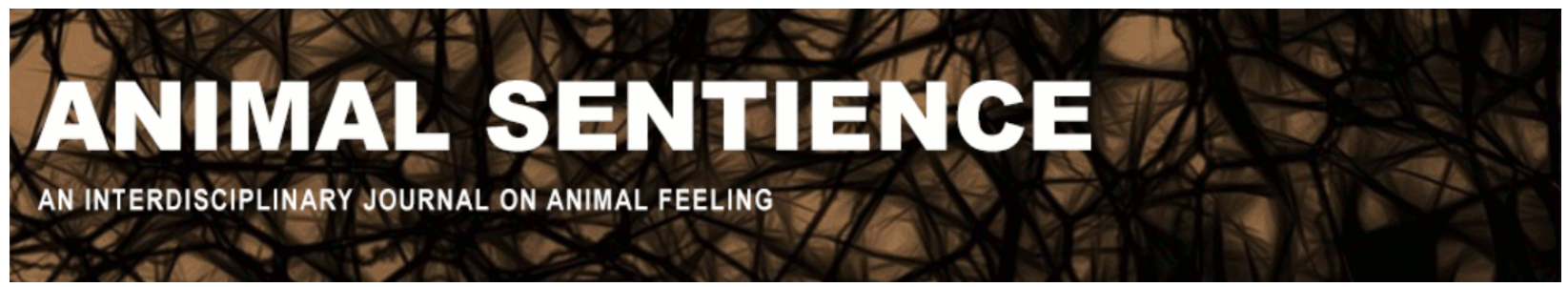

Franklin, Robert G., Jr. (2019) Why are sheep sheepish? How perception affects animal stereotyping. Animal Sentience 25(15)

DOI: $10.51291 / 2377-7478.1448$

Date of submission: 2019-05-17

Date of acceptance: 2019-05-21

(c)

This article has appeared in the journal Animal

Sentience, a peer-reviewed journal on animal

cognition and feeling. It has been made open access,

free for all, by WellBeing International and deposited

in the WBI Studies Repository. For more information,

please contact

wbisr-info@wellbeingintl.org.

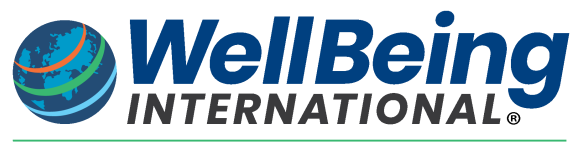

SOLUTIONS FOR PEOPLE, ANIMALS AND ENVIRONMENT 


\title{
Why are sheep sheepish? How perception affects animal stereotyping
}

Commentary on Marino \& Merskin on Sheep Complexity

\author{
Robert G. Franklin, Jr. \\ Department of Behavioral Sciences, Anderson University, Anderson, SC
}

\begin{abstract}
Marino \& Merskin present compelling evidence that many stereotypes of sheep are incorrect. One factor that may play an important role in animal stereotyping is the physical appearance of animals, which can directly lead to stereotyping through automatic mental processes. Sheep have a round and babyish appearance that directly evokes judgments of warmth and docility. Depictions of sheep in art, and especially cartoons, reinforce this stereotype.
\end{abstract}

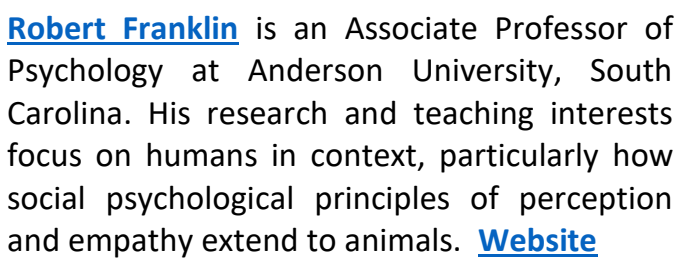

Robert Franklin is an Associate Professor of Psychology at Anderson University, South Carolina. His research and teaching interests focus on humans in context, particularly how and empathy extend to animals. Website

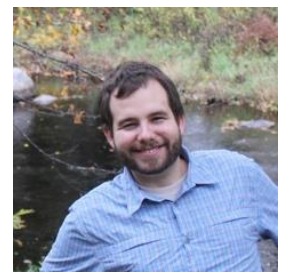

Marino \& Merskin (2019) (M\&M) provide an excellent overview of the cognition of sheep, with many examples dispelling the stereotypes of sheep as passive and unintelligent creatures. The evidence in this article shows that sheep have advanced cognition and impressive social capabilities, both as individual animals and as part of a collective social organization.

These findings contradict common stereotypes of sheep. As M\&M discuss, sheep are frequently described as weak, docile, and followers. The words "sheepish" and the newer term "sheeple" illustrate this stereotype. Many cultural and historical reasons can explain how incorrect stereotypes develop. Here, I will discuss how perceptual processes in the visual system can create and sustain the stereotype of sheep.

Stereotyping is an important process of social cognition where humans attribute a set of characteristics to members of a certain group, often automatically (e.g., Devine, 1989). Most of the research on stereotyping examines how humans stereotype other human groups, but these processes extend to animal species. According to the stereotype content model, stereotyping in humans and animals leads to judgments in two dimensions: dominance and warmth (Sevillano \& Fiske, 2015). Dominance reflects a judgment about an individual's ability and power whereas warmth reflects a judgment about an individual's intent, good or bad.

Though Sevillano \& Fiske did not examine sheep in their study, it is very likely that sheep would be thought of as high in warmth and low in competence, which is consistent with many farm animals such as cows, pigs, and rabbits. Sheep are often portrayed as having low power and are almost always placed in a submissive role in stories. Sheep are also seen as morally pure or clean examples, especially in the Judeo-Christian context. The Torah called for sacrificing a lamb as atonement for sin, and this image of purity was adapted into Christian belief as well, as Jesus was referred to as the "Lamb of God". One example of this is the New Testament story where 
Jesus describes judgment as separating the sheep from the goats, where the sheep are given eternal rewards while the closely related goats are punished.

Physical appearance directly leads to judgments that can form the basis of stereotypes. According to ecological psychology, perception is disposed to recognize cues that are most relevant for survival. Two of the most important cues of survival are recognizing danger and safety, which are strongly related to dominance and warmth. One of the primary cues for this is recognizing angularity versus roundness in an object or creature. Angular cues are often threatening, such as jagged rocks or sharp teeth, whereas rounder cues indicate safety (Franklin, Adams, Steiner, \& Zebrowitz, 2019). As sheep are very round and soft, they elicit immediate judgments of warmth and friendliness (Figure 1). This is especially the case as many sheep breeds are naturally polled, or hornless.

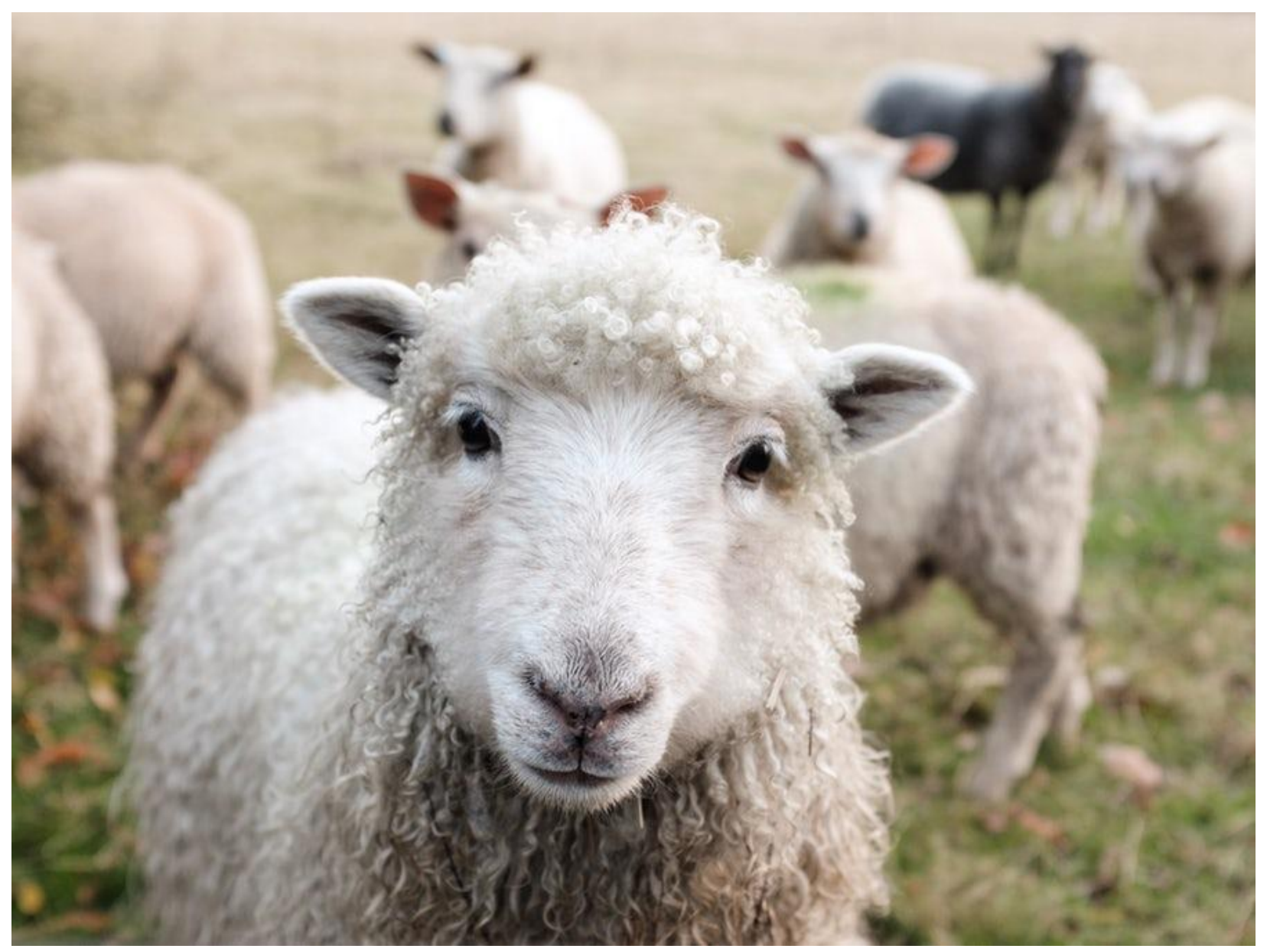

Figure 1. Image of sheep (public domain image).

A second cue of physical appearance which may lead to stereotypes is the presence of babyish cues in the faces of animals. Babyish cues, such as large eyes and a round face, lead to perceptions of high warmth and low competence in both human and animal faces (e.g., Berry \& McArthur, 1985). One reason for this is the baby schema (kindchenschema) as proposed by Lorenz (1943), or the idea that the presence of babyish features leads to caretaking behavior in animals and 
humans. Adult sheep often have a roundish appearance while lambs have large, wide-set eyes that additionally convey youth. Babyish features may explain why lambs are seen as particularly innocent and vulnerable.

A notable exception to this stereotype that helps to validate the importance of visual cues is that rams are often an exception to the stereotype of sheep. In the US, a popular model of truck, a football team, and several college teams are named after rams, with aggressive logos. Bighorn sheep are a species of sheep with large horns. Though bighorn sheep are closely related to domestic sheep and able to interbreed, they do not have the same stereotype. Thus, bighorn sheep can be seen as an example of subtyping, where a member of a group violates a stereotype. When some members of a group violate a stereotype, they do not change the stereotype overall but are seen as exceptions that prove the rule. In this case, bighorn sheep are seen as particularly aggressive for a sheep.

Physical appearance is only one reason for the stereotyping of sheep, but it plays a very important role in culture, especially for those who do not interact with domestic sheep. Many children learn about sheep through cartoons, where sheep are illustrated with highly caricatured roundness and babyish features (Figure 2). In order to help combat these stereotypes and argue for the ethical treatment of animals based on their cognitive traits, visual depictions of animals should not evoke judgments that reinforce the incorrect stereotypes, as M\&M describe.

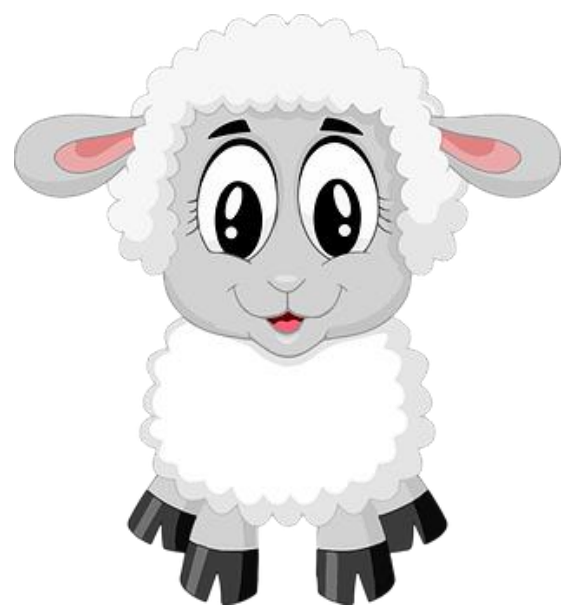

Figure 2. Cartoon image of sheep (public domain image). 


\section{References}

Berry, D. S., \& McArthur, L. Z. (1985) Some components and consequences of a babyface. Journal of Personality and Social Psychology, 48(2), 312-323.

Devine, P. G. (1989) Stereotypes and prejudice: Their automatic and controlled components. Journal of Personality and Social Psychology, 56(1), 5-18.

Franklin, R. G., Jr., Adams, R. B., Jr., Steiner, T. G., \& Zebrowitz, L. A. (2018) Reading the lines of the face: Angularity and roundness in perceiving facial anger and joy. Emotion.

Lorenz, K. (1943) Die angeborenen formen möglicher erfahrung. Zeitschrift für Tierpsychologie, 5(2), 235-409.

Marino, L., \& Merskin, D. (2019) Intelligence, complexity, and individuality in sheep. Animal Sentience 25(1).

Sevillano, V., \& Fiske, S. T. (2016) Warmth and competence in animals. Journal of Applied Social Psychology, 46(5), 276-293. 